\title{
Bio-Wein Konsum in Deutschland
}

\author{
Gergely Szolnoki und Eva Christ \\ Geisenheim University, Institut für Betriebswirtschaft und Marktforschung, Von-Lade-Str. 1, 65366 Geisenheim, Germany
}

\begin{abstract}
Die Thematik "Bio" hat Einzug in das alltägliche Leben der Gesellschaft genommen. Lebensmitteleinzelhandel und Discounter erkennen diesen Trend und folgen der Nachfrage der Konsumenten nach ökologisch erzeugten Produkten. Von diesem Wandel ist augenscheinlich auch die deutsche Weinwirtschaft betroffen. Doch stellt sich in diesem Zusammenhang die Frage, ob Lebensmittel- und Weinmarkt hinsichtlich der Nachfrage nach biologisch erzeugten Produkten, einander gleichzustellen sind. Mithilfe einer repräsentativen Befragung von 2.000 Personen aus Deutschland wurden die Biowein-Konsumenten detailliert beschrieben und nach Kriterien wie Alter, Geschlecht und sozialer Klasse aufgeteilt. Bei der Segmentierung stellte sich heraus, dass die Biowein-Käufer vermehrt aus der Oberschicht stammen, somit gebildeter sind und ein höheres Nettoeinkommen haben. Ebenfalls konsumieren sie häufiger Wein, präferieren sie die Geschmacksrichtung „trocken“ und kaufen Weinen auch aus dem höheren Preissegment. Darüber hinaus besitzen sie ein höheres Interesse und Wissen für bzw. an Wein.
\end{abstract}

\section{Einleitung}

Ein vielseitiges Angebot an Bio-Lebensmitteln in kommerziellen Einkaufsstätten hat dazu geführt, dass Konsumenten von Bio-Lebensmitteln kein klares Zielgruppenprofil zugeordnet werden kann. So verhält es sich auch bei Wein. Wie wird sich der deutsche Bio-Weinmarkt weiter entwickeln und welche Maßnahmen müssen getroffen werden, um diesen zu stabilisieren? Ein wichtiger Grundbaustein in diesem Prozess ist ein Zielgruppenprofil, welches in dieser Arbeit ermittelt werden soll, um mehr Transparenz in den deutschen Bio-Weinmarkt zu bringen.

Mithilfe einer repräsentativen Befragung soll es ermöglicht werden, den Bio-Weinkonsumenten in eine klare Segmentierung einordnen zu können. In diesem Zusammenhang stellt sich ebenfalls die Frage: Welche Auswirkungen hat der Bio-Trend auf den deutschen Weinmarkt? Welche Konsequenzen müssen getroffen werden, um auf Veränderungen im Bio-Weinmarkt reagieren zu können? Wer ist der Bio-Weinkonsument und welche Erwartungen hat er an das Produkt?

\section{Literaturüberblick}

Im folgenden Kapitel wird der Markt für Bio-Weine näher beleuchtet, da sich dieser erheblich von der allgemeinen Struktur der Bio-Branche unterscheidet und einen alleinstehenden Bereich darstellt. Das oben $\mathrm{zu}$ erkennende Wachstum der Bio-Branche trifft auf die Wein-Branche in anderem Ausmaß zu und ist nicht mit diesem gleichzustellen. Betrachtet man die Verteilung der regelmäßig gekauften Produkte mit Bio-Siegel, zeigt sich, dass Getränke im Vergleich zu anderen Bio-Produkten, lediglich ein Präferenzniveau von 2,4\% und Wein eine Präferenz von $3,7 \%$ erreichen. Somit sind Bio-Weine als Nischenprodukt einzuordnen und stehen nicht im
Fokus der allgemeinen Bio-Orientierung der Verbraucher. (Hoffmann und Szolnoki, 2010)

Zudem unterscheidet sich Wein von anderen Lebensmitteln durch eine sehr große Sortimentstiefe, nicht nur im Fachhandel, sondern auch im Lebensmitteleinzelhandel. Dies liegt nicht nur an der Vielzahl der Erzeuger und Anbieter, sondern auch an den sehr heterogenen Präferenzen der Verbraucher (Goldstein et al., 2008)

Wein ist im Gegensatz zu vielen anderen Lebensmitteln ein Genussmittel, bei dem der Geschmack eine außerordentliche Rolle spielt. Der Geschmack hängt wiederum von einer Vielzahl von Faktoren ab, wie Terroir, Rebsorte, Herkunftsland, Jahrgang. Als Folge sind Verbraucher zum Zeitpunkt des Kaufes meist nicht in der Lage, den Geschmack des Weines allein anhand der Angaben auf der Flasche zu beurteilen (Lockshin et al., 2006).

Seit den 90er Jahren wächst die Bedeutung des BioMarktes in Europa kontinuierlich. Von dem Bio-Boom betroffen sind auch die Bio-Weinbranche und der BioRebbau (Willer und Yussefi, 2006). Dies sagt zwar aus, dass auch die Weinbranche einen Zuwachs von BioWeinen $\mathrm{zu}$ verzeichnen hatte und dass dieser Zuwachs von dem des allgemeinen Bio-Marktes beeinflusst wurde. Jedoch ist es fraglich, ob wirklich von einem "Boom" in der Weinwirtschaft gesprochen werden kann. Da die Maßstäbe des deutschen Weinmarktes in weitaus höheren Dimensionen liegen als die des jetziges Bio-Weinbaus, der nur ca. 6,0\% des gesamten deutschen Weinmarkts ausmacht.

Betrachtet man die Verteilung der globalen ÖkoRebfläche nach Kontinenten, liegt Europa mit 89,1\% auf Platz eins. Es folgen Nordamerika (4,5\%), Asien $(3,2 \%)$ Südamerika $(3,0 \%)$, Ozeanien $(0,2 \%)$ und Afrika mit $0,1 \%$. Der Anteil der gesamten Bio-Rebfläche 
weltweit liegt bei ca. $2 \%$ und beträgt 284.265 ha. Die Verteilung der globalen Öko-Rebfläche nach Ländern, in Abbildung $3 \mathrm{zu}$ sehen, ordnet sich wie folgt: Spanien ist das Land mit der größten Öko-Rebfläche und macht 30,4\% der weltweiten Öko-Rebfläche aus. Es folgen Frankreich $(23,5 \%)$ und Italien (20,3\%). Durch das frühzeitige Erkennen des Bedarfs an ökologisch produziertem Wein erfolgte in Italien eine entsprechende Umorientierung der Produktion. Auch für die französische Produktion ist ein hohes Exportvolumen des ökologisch produzierten Weins bekannt. Dabei stehen Deutschland und die skandinavischen Staaten als Hauptabsatzgebiete im Vordergrund (Fader und Porten, 2009).

In Deutschland haben sich die Anbauflächen im Bio-Weinbau in den vergangenen fünf Jahren mehr als verdoppelt. So stieg die Rebfläche im Jahr 2012 auf 7.400 ha an, was einen Anteil von 7,6\% an der gesamten deutschen Rebfläche (102.000 Hektar) ausmachte. (BÖLW, 2014) Insgesamt gibt es 18.700 Weinbaubetriebe in Deutschland, davon sind 700 BioWeingüter. Die Bio-Weinbaubetriebe machen somit 3,7\% der gesamten Weinbaubetriebe in Deutschland aus. (Wechsler, 2014).

Führendes Bundesland beim Ökoweinbau ist Rheinland-Pfalz: dort hat sich die ökologisch bewirtschafteten Rebfläche von 1.751 Hektar im Jahr 2006 auf 4.861 Hektar im Jahr 2013 mehr als verdoppelt. Die Zahl der zertifizierten Öko-Weinbaubetriebe in Rheinland-Pfalz stieg von 184 im Jahr 2006 auf 379 im Jahr 2012. Im Jahr 1980 liegen die Anfänge des rheinland-pfälzischen Ökoweinbaus, zu dieser Zeit gab es ganze fünf Betriebe mit rund 38 Hektar Rebfläche, die nach ökologischen Kriterien bewirtschaftet wurde (GW, 2014).

Heute wird der Ökoweinbau von den Bundesländern subventioniert. Beispielsweise Rheinland-Pfalz fördert den ökologischen Weinbau mit dem Programm-AgrarUmwelt-Landschaft (PAULa) mit $800 € /$ ha in der Umstellungsphase, zwei Jahre lang. $680 € /$ ha nach der Umstellung und $35 € /$ ha Kontrollkostenzuschuss der bis maximal 530 €ausgeschöpft werden kann (dlr-rlp, 2009).

Welche Intention steckt hinter dem Entschluss, eine konventionell bewirtschaftete Rebfläche auf eine ökologische umzustellen? Die drei Ausgangsmöglichkeiten sind: die eigene persönliche Entscheidung, das Wohl der Umwelt und die Zufriedenstellung des Kunden. Man könnte viele individuelle Entscheidungen von unterschiedlichen Erzeugern in zwei Kategorien aufteilen: auf der einen Seite stehen qualitätsorientierte Betriebe, die aus internen Überlegungen und aus Überzeugung auf eine ökologische Wirtschaftsweise umsteigen. Hier steht eine klare Unternehmensphilosophie im Vordergrund. Andererseits waren hohe Fassweinpreise sowie die staatliche Förderung für Bio-Wein ein Grund für eine ökologische Umstellung und die Anpassung an die Veränderung am Markt. Fraglich ist im letzteren Fall die Verankerung der Nachhaltigkeit. Die irrtümliche Annahme, mit einem ökologisch bewirtschafteten Weingut wirtschaftlichen Erfolg zu generieren, zeigt ebenfalls eine Studie (Mend, 2013), die einen Kennzahlenvergleich von ökologischen und konventionellen Weinbaubetrieben durchführte. Die Ergebnisse zeigten, dass der ökonomische Erfolg der
Weinbaubetriebe weiterhin in einer klaren, zielstrebigen Unternehmensführung liegt und nicht von dem gewählten Wirtschaftsansatz abhängig ist.

Der größte Anteil der Bio-Weinbaubetriebe tritt einem Öko-Verband bei. Hier ist folgende Verteilung der Betriebe festzustellen, allerdings ist in Abbildung $5 \mathrm{zu}$ beachten, dass hier die EU-zertifizierten Weinbaubetriebe nicht aufgeführt sind, da deren Anzahl nicht bekannt ist, jedoch ist die Fläche der EU-zertifizierten Betriebe auf 2.730 Hektar zu beziffern. ECOVIN, bei dem ausschließlich Öko-Weingüter vertreten sind, ist der Verband mit den meisten Bio-Weingütern, und somit auch mit der größten Öko-Rebfläche. Es folgt der Verband Bioland mit 126 Bio-Weingütern und 1025 Hektar. Der Verband Deutscher Prädikatsweingüter (VDP), eigentlich kein Verband für Öko-Betriebe, ist ebenfalls hier erwähnt, da deren BioWeingüter keinen zusätzlichen Verbänden beitreten und somit ebenfalls erfasst werden können. 49 Betriebe des VDPs sind Bio-Weingüter, die insgesamt eine Fläche von 980 Hektar ökologisch bewirtschaften. Somit arbeitet ein Viertel des VDPs nach ökologischen Richtlinien, Tendenz steigend (VDP, 2014). Die weiteren Verbände wie demeter, Naturland oder Biokreis weisen vergleichsweise geringere Zahlen an beigetretenen Weinbau-Betrieben auf. Dies ist wahrscheinlich darauf zurückzuführen, dass diese Verbände nicht auf Weingüter spezialisiert sind, sondern allgemein für eine ökologische Landwirtschaft stehen.

Führt man eine Modellrechnung durch, die die deutsche Bio-Weinproduktion darstellen soll, kommt man mit folgender Rechnung auf ein Ergebnis in Millionen Hektolitern.

Man multipliziert die gesamte deutsche Bio-Rebfläche, also 8000 Hektar, mit dem Durchschnittsertrag eines Bio-Betriebes, der 70 Hektoliter (Lorenz, 2014) pro Hektar beträgt. Man kommt auf einen Betrag von 560.000 Hektolitern. Addiert man die Produktion der deutschen Bio-Betriebe mit der Zahl der Importe, also ca. 600,000 Hektoliter (Römmelt, 2015), kommt man auf 1,16 Mio. Hektoliter. Im Vergleich dazu, ist der gesamte deutsche Weinmarkt, inklusive Importe, mit circa 17,7 Millionen Hektolitern, ohne Vorräte und ohne Schaumwein zu beziffern. Daraus lässt sich schließen, dass der deutsche Bio-Weinmarkt 6,6\% vom gesamten deutschen Stillweinmarkt ausmacht.

Ebenfalls erkenntlich aus diesen Zahlen wird der ProKopf-Konsum der Deutschen. Rechnet man mit einer deutschen Bevölkerung ab 16 Jahren, also 65,7 Mio. Menschen, kommt man auf einen Pro Kopf Verbrauch von 1,4 Litern Bio-Wein.

\section{Vorgehensweise}

Dieses Kapitel widmet sich der methodischen Vorgehensweise des Projektes. Im Mai 2014 wurde eine Mehrheitsumfrage zu Wein, Bio-Wein, Sekt und anderen Getränken durchgeführt. Grundlage dieser Umfrage stellt eine zufällig ausgewählte Stichprobe von 2050 Personen dar, die repräsentativ für die soziodemografische Struktur der Bevölkerung ab 16 Jahren stehen. Mit Hilfe eines vollstrukturierten Fragebogens wurden verschiedene Fragen zur Nutzung, zum Einkauf und zur Bewertung 


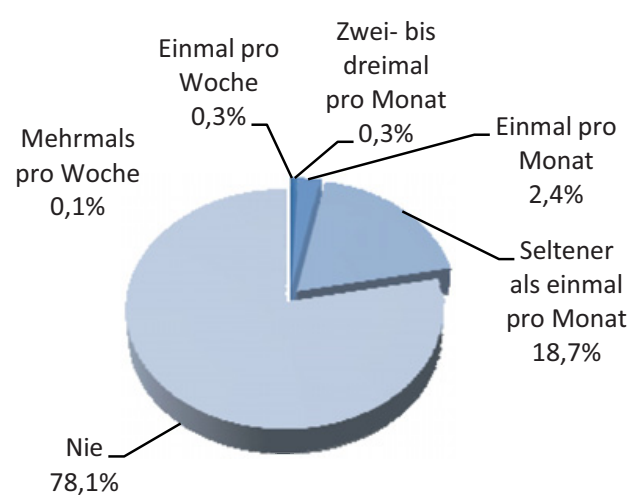

Abb 1. Kaufhäufigkeit von Bio-Wein der Weintrinker.

von Wein gestellt. Die einzelnen Fragestellungen werden im Zusammenhang mit der Darstellung der Ergebnisse erläutert.

Die Auswahl der Testpersonen wurde auf der Basis einer Quotenstichprobe aus einer Grundgesamtheit von 63,7 Millionen Personen, die durch 500 Interviewer persönlich und vor Ort befragt wurden, durchgeführt. Die Felderhebung zu dieser Studie führte die Gesellschaft für Konsumforschung (GfK-Omnibussystem) in Nürnberg durch.

\section{Ergebnisse}

\subsection{Konsumstruktur der Bio-Weintrinker}

In Abbildung 1 wurden die Antworten auf die Frage dargestellt, wie oft die Befragten regelmäßig Bio-Wein kaufen. Dabei wurden nur die Weintrinker unter den Befragten beachtet.

Dabei stellte sich heraus, dass 22\% der Weintrinker, dies würde also 8,9 Millionen Menschen in Deutschland über 16 Jahren repräsentieren, ab und an bewusst BioWein kaufen. Es wurden also die Antwortmöglichkeiten "mehrmals pro Woche" bis "seltener als einmal im Monat zusammengefasst". Schlussfolgernd kaufen $78 \%$ der Weintrinker, repräsentativ für 31,8 Millionen Deutsche über 16 Jahre, keinen Bio-Wein. In diesem Kapitel der Konsumstruktur werden diese beiden Gruppen einander gegenübergestellt. Der Vergleich zwischen den Bio-Weinkäufern und den Nicht-Bio-Weinkäufern wird Aufschluss darüber geben, inwieweit sich Bio-Weinkäufer von den durchschnittlichen Weintrinkern unterscheiden und welche Präferenzen sie in verschiedenen Kategorien an den Tag legen.

Die grundsätzliche Einstellung zu Wein wurde mithilfe zweier Fragestellungen erhoben, denen die Befragten auf einer Skala von -3 bis +3 ablehnen oder zustimmen konnten. So bedeutet -3 , dass die Aussage "voll und ganz abgelehnt" wurde und +3 "voll und ganz zugestimmt". Die in Abbildung 2 dargestellten Werte entsprechen den Mittelwerten der Aussagen. Betrachtet man zunächst die Verteilung der Interessenausprägung bei Wein, ist festzustellen, dass der Durchschnitt der Bio-Weinkäufer einen Wert von 0,17 ergibt. Jedoch ist der Durchschnittswert der normalen Weinkäufer bei weitem mehr dem negativen Bereich zugewandt. Somit ist

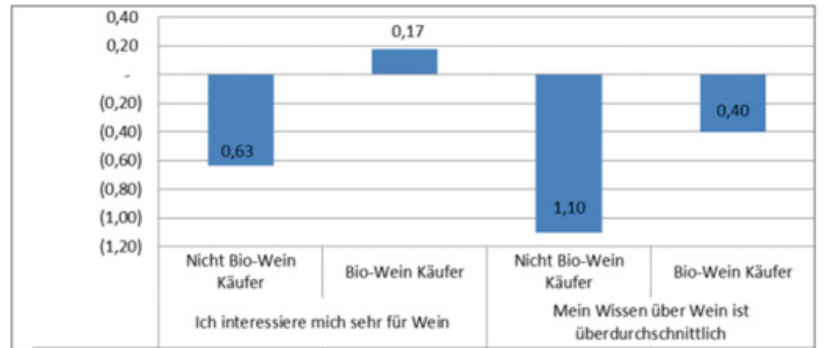

Abb 2. Verteilung der Interessen- und Wissensausprägung.

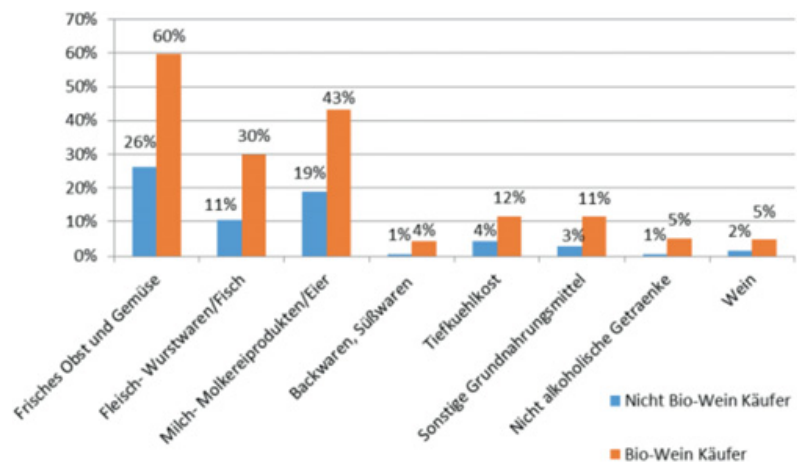

Abb 3. Regelmäßiger Kauf von Produkten mit Bio-Siegel.

festzuhalten, dass die Bio-Weinkäufer nur im Vergleich betrachtet, ein höheres Interesse für Wein aufbringen als normale Weinkonsumenten. Dies ist wiederum auf die Wahl der Einkaufsstätte zu beziehen. Da Bio-Weinkäufer ein größeres Interesse für Wein aufbringen, kaufen sie auch eher Wein im Fachhandel, als die normalen Weinkäufer.

Die Einschätzung über das Wissen von Wein, ist auch bei den Bio-Weinkäufern in einem negativen Bereich von $-0,4$. Jedoch gewinnt diese Zahl an neuer Bedeutung vergleicht man sie mit den Nicht-Bio-Weintrinkern, die einen Durchschnittswert von $-1,1$ erreichen. Somit hat der Bio-Weintrinker ein höheres Wissen bezüglich Wein und ist interessierter.

Eine weitere Frage bezüglich Bio-Lebensmittelmittel, war "Welche der folgenden Produkte kaufen Sie regelmäBig mit Bio-Siegel?" (Abb. 3). Hier waren Mehrfachantworten zugelassen. Bei dieser Frage zeichnet sich ab, dass Bio-Weinkäufer öfter zu Bio-Produkten greifen als NichtBio-Weinkäufer. So geben $60 \%$ der Befragten an, frisches Obst und Gemüse mit Bio-Siegel zu kaufen, während dies nur 26\% der Nicht-Bio-Weinkäufer tun. Bio-Obst und Gemüse ist die am häufigsten gekaufte Produktgruppe. Am zweithäufigsten kaufen die Bio-Weinkonsumenten Milchund Molkereiprodukte und Eier. Hier geben $43 \%$ an, diese regelmäßig zu kaufen, während nur zu 19\% der NichtBio-Weintrinker auf diese Bio-Produkte zurückgreifen. An dritter Stelle stehen Fleisch- und Wurstwaren mit Bio-Siegel: $30 \%$ der Bio-Weinkäufer geben an diese zu kaufen, während nur $11 \%$ der normalen Weinkonsumenten regelmäßig zu dieser Produktgruppe greift.

Allgemein ist anhand dieses Graphen festzustellen, dass die Bio-Weinkäufer alle Bio-Produktgruppen häufiger regelmäßig kaufen als die Nicht-Bio-Weinkäufer. Dies lässt schließen, dass der Kauf von Bio-Lebensmitteln mit 


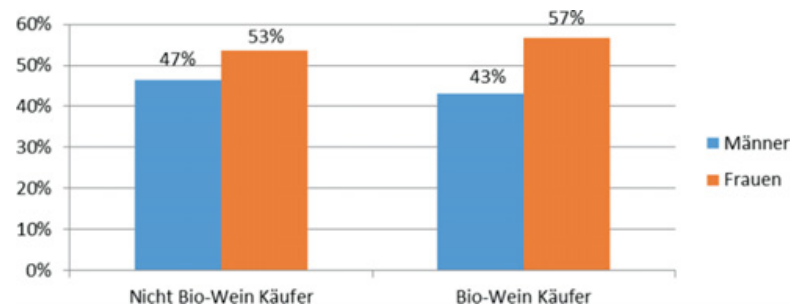

Abb 4. Geschlechtsverteilung der Bio-Weinkäufer (in \%).

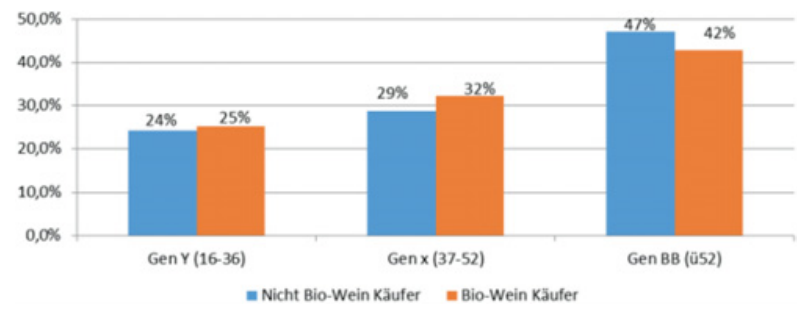

Abb 5. Altersgruppen der deutschen Bio-Weinkäufer.

dem Kauf von Bio-Wein einhergeht. Zumindest besteht ein Zusammenhang zwischen beiden Produktgruppen. So sind Konsumenten, die regelmäßig Bio-Lebensmittel kaufen, eher dazu bereit auch Bio-Wein zu kaufen.

\subsection{Segmentierung der Bio-Weinkäufer}

Bei der Segmentierung nach Geschlecht ist zu beachten, dass der Anteil der weiblichen Bio-Weinkäufer mit 57\% eine stärkere Bedeutung einnimmt, als der der Männer mit einem Anteil von 43\% (Abb. 4).

Altersgruppen haben bei Konsumgütern einen starken Einfluss auf die Struktur der Nachfrage. Gerade, weil die Wünsche und Bedürfnisse von Konsumenten sich mit deren Alter ändern (Armstrong et al., 2011)

Die Einteilung in drei Altersgruppen (Abb. 5) ermöglicht es, wesentliche Unterschiede zwischen den einzelnen Altersphasen kenntlich $\mathrm{zu}$ machen und unterschiedliche Verhaltensweisen der einzelnen Gruppen herauszustellen.

Die hier verwendete Einteilung erfolgte in die international übliche Unterteilung: Generation Y, Generation X und Babyboomer. Die Generation Y ist definiert als die Altersgruppe bis 35 Jahre. Diese Altersgruppe ist bei den Bio-Weinkäufern die am wenigsten vertretene mit $25 \%$. Dies ist damit zu begründen, dass bei dem Konsumeinstieg in alkoholische Getränke, jüngere Personen erst deutlich nach dem 20. Lebensjahr dem Wein näher kommen und erst nachfolgend ihre Konsumintensität steigern (Hoffmann und Szolnoki, 2014). Daher ist die Generation $\mathrm{X}$, Personen von 37-52 Jahre bei den Bio-Weinkäufern mit $32 \%$ vertreten und alle Personen über 52 Jahre mit $43 \%$.

Die Abgrenzung der Regionen folgt der in der kommerziellen Marktforschung häufig verwendeten Unterteilung der Bundesrepublik Deutschland in folgende Regionen: - Nord-West beinhaltet die Bundesländer Schleswig-Holstein, Hamburg, Bremen und Niedersachsen. - NRW steht für das Bundesland NordrheinWestfalen. - Mitte-West schließt die Bundesländer Hessen, Rheinland-Pfalz und das Saarland ein. - Nordost beinhaltet die Bundesländer Mecklenburg-Vorpommern

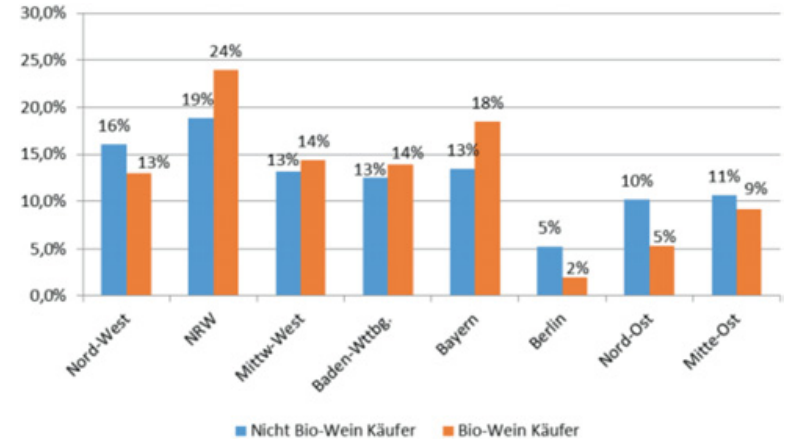

Abb 6. Geographische Herkunft der Bio-Weinkäufer nach Nielsen-Regionen (in \%).

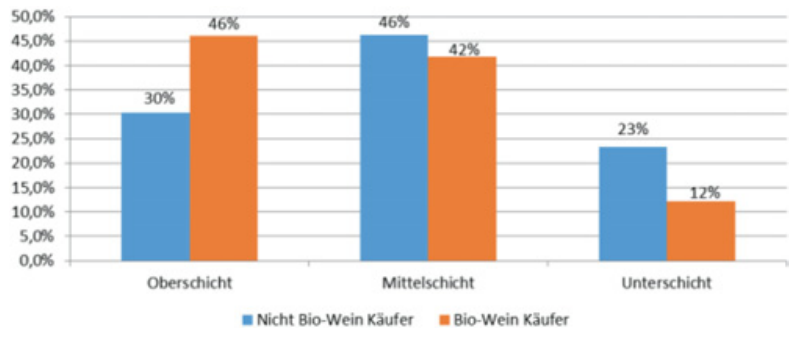

Abb 7. Verteilung der sozialen Klassen der Bio-Weinkäufer.

und Brandenburg, während - Mitte-Ost die Bundesländer Sachsen, Sachsen-Anhalt und Thüringen beinhaltet.

Herausstechend bei dieser Abbildung ist, dass die Bio-Weinkäufer vermehrt aus Nordrhein-Westfalen (24\%) und Bayern (19\%) stammen. Während sich die NichtBio Weinkäufer gleichmäßiger auf die einzelnen Regionen verteilen. Besonders wenig vertreten sind die BioWeintrinker in Berlin mit $2 \%$ und in der Region Nord-Ost mit 5,3\% (Abb. 6).

Die sozialen Schichten, die im Zentrum des nächsten Punktes stehen, wurden unter Betrachtung des Nettoeinkommens, des Bildungsabschlusses und des derzeitigen Berufes der Befragten gebildet. So ergaben sich drei Klassen: Ober-, Mittel- und Unterschicht.

Die Verteilung der sozialen Klassen der BioWeinkäufer wurde hier erneut im Vergleich zu den Nicht-Bio-Weinkäufern ausgewertet (Abb. 7). Deutlich zu erkennen ist, dass die Bio-Weinkäufer mit $46 \%$ stärker in der Oberschicht vertreten sind, als die Nicht-BioWeinkäufer mit 30\%. In der Mittelschicht verteilen sich beide Käuferschichten gleichmäßiger, auch wenn die Bio-Weinkäufer hier etwas weniger mit $42 \%$ vertreten sind. Am wenigsten sind die Bio-Weinkäufer in der Unterschicht mit nur $12 \%$ vertreten. Dies zeigt, dass die Bio-Weinkäufer am häufigsten aus der Oberschicht stammen, was vor allem der Vergleich mit den Nicht-BioWeinkäufern vor Augen führt.

\section{Fazit}

Deutschland stellt den größten Markt für Bio-Lebensmittel in Europa dar, was vor Augen führt, dass wir uns im Zentrum der Veränderung befinden und weiterhin als Vorreiter gelten können. Daraufhin wurde ebenfalls festgestellt, dass die deutschen Haushalte immer mehr 
Geld für Bio-Lebensmittel ausgeben, was somit ein wichtiger Hinweis auf dem Wachstum dieser Branche kennzeichnet.

Die Analyse des deutschen Bio-Weinmarktes hat gezeigt, dass das Produkt Bio-Wein nicht mit den herkömmlichen Bio-Lebensmitteln vergleichbar ist. Auf Grund dessen wurde ein separates Bild des BioWeinmarktes geschaffen, was zeigte, dass Potenzial für die Zukunft vorhanden ist, welches jedoch nur mithilfe von weiteren Marketingmaßnahmen ausgeschöpft werden kann.

Dabei spielen die Konstellation Markt, Betriebe, Verbände und Konsumenten eine entscheidende Rolle. Die Analyse der deutschen Bio-Weinkonsumenten zeigte deutlich durch einen Vergleich der Konsumstruktur, dass sich Bio-Weinkäufer von Nicht-Bio-Weinkäufern unterscheiden, was die Bio-Weinkäufer als allein stehende Konsumentengruppe identifizierte.

Die darauffolgende Segmentierung der Bio-Weinkäufer spaltete deren Präferenzen noch einmal abhängig von Alter, Geschlecht, Herkunft und sozialer Klasse auf und veranschaulichte dadurch die einzelnen soziodemographischen Eigenschaften. Dabei stellte sich heraus, dass die Bio-Weinkäufer vermehrt aus der Oberschicht stammen, somit gebildeter sind und ein höheres Nettoeinkommen haben. Ebenfalls konsumieren sie häufiger Wein, was sie für Präferenzen von Farbe des Weines, Geschmacksrichtungen, Einkaufsstätte sensibilisiert und ihr Interesse und Wissen an Wein steigert.

Ebenfalls erkenntlich aus der repräsentativen Studie wurde, dass Bio-Weinkäufer auch häufiger BioLebensmittel kaufen, was zeigt, dass beide Produktgruppen miteinander verbunden sind, obwohl sich Wein deutlich von Lebensmitteln unterscheidet.

Deutlich wurde ebenfalls in dieser Arbeit, dass sich der deutsche Bio-Weinmarkt mitten in einem Prozess befindet, der noch einige Arbeit an dem Marketingkonzept Bio-Wein fordert. Die weitere Entwicklung der BioWeinbranche gestaltet sich somit abhängig von dem Reagieren und Agieren der Betriebe, Weingüter und Verbände. Obwohl dies von vornherein zu erahnen war, soll betont werden, dass der analysierte BioWeinkonsument eine klare Vorstellung von Bio-Wein verinnerlicht haben muss, um gezielt und willentlich $\mathrm{zu}$ dem Produkt Bio-Wein zu greifen.

\section{Literatur}

G. Armstrong, V. Wong, J. Saunders, P. Kotler. Grundlagen des Marketings Pearson Studium, München (2011)

BÖLN. Bundesprogramm ökologischer Landbau und andere Formen nachhaltiger Landwirtschaft Präferenzen und Zahlungsbereitschaft deutscher Verbraucher bei Öko-Wein; www. bundesprogrammekolandbau.de/forschungsmanagement/projekt liste/oekonomie/ eingesehen am 15.10.2014 (2014)

BÖLN. Bundesprogramm ökologischer Landbau und andere Formen nachhaltiges Landwirtschaft -
Ökobarometer 2013 Repräsentative Bevölkerungsbe fragung im Auftrag des Bundesministeriums für Ernährung, Landwirtschaft und Verbraucherschutz (BMELV); http://www.bmel.de/SharedDocs/ Downloads/Ernaehrung/Dekobarometer_2013. pdf ; jsessionid=B62BA9DD31C535248131F69 EB461042B.2_cid385?__blob=publicationFile eingesehen am: 11:10.2014 (2013)

BÖLW. Bund ökologische Lebensmittelwirtschaft - Zahlen, Daten, Fakten 2014;www. boelw.de/ zahlendatenfakten.html\#c3014, eingesehen am 10.10.2014 (2014)

DWI. Deutsches Weininstitut - Öko-Weinbau in Deutschland;www. deutscheweine.de/icc/Internet-DE/ med/dd7/dd7, eingesehen am 29.09.2014 (2013)

DLR-RLP. Dienstleistungszentren ländlicher Raum Rheinland-Pfalz - Weinmarketing; http://www.dlr . rlp.de/Internet/global/themen.nsf/0/9e1c4 cb8696b79c1c1257632002c9ead?OpenDocument eingesehen am 20.09.2014 (2009)

B. Fader, M. Porten. Bundesministerium für Ernährung, Landwirtschaft und Verbraucherschutz Marktentwicklung und Vermarktung im Öko-Weinbau, http://www. oekolandbau.de/fileadmin/redak tion/oeko_lehrmittel/Fachsschulen_Agrar/ Weinbau/fwb_modul_c/fwb_c_01/fwbmc01_06_ 2009.pdf eingesehen am 06.10.2014 (2009)

R. Goldstein, J. Almenberger, A. Dreber, J.W. Emerson, A. Herschkowitsch, J. Katz. Do More Expensive Wines Taste Better? http: //www . darkcoding. net/research/wine\% 20economics_Robin\%20Goldstein_vol\%2031.pdf eingesehen am 14.10.2014 (2008)

Gourmet Welten. Biologischer Weinanbau in Deutschland; http://www.nikos-weinwelten.de/beitrag/ biologischer_weinanbau_in_deutschland/, eingesehen am 10.10.2014 (2014)

D. Hoffmann, G. Szolnoki. Wie ticken die Verbraucher bei Bio? Weinwirtschaft, 18/2010, 40-46 (2010)

D, Hoffmann, G. Szolnoki. Online, face to face and Telephone Surveys - Comparing Different Sampling Methods in Wine Consumer Research. Wine Economics and Policy http://www. sciencedirect.com/science/article/pii/ S2212977413000331 am 08.11.2014 (2014)

D, Hoffmann, G. Szolnoki. Neue WeinkundenSegmentierung in Deutschland. Gesellschaft zur Förderung der Hochschule Geisenheim e.V. (2014)

L. Lockshin, W. Jarvis. Sensitivity Analysis of Purchase Cues for Wine Using a Discrete Choice Experiment; http://mintinnovation.com/links/docs/ conjoint_analysis/CBC $\% 20$ on $\% 20$ Wines.pdf eingesehen am 13.11.2014 (2006)

M. Mend. Wettbewerbsfähigkeit Bio-Weingüter. Landwirtschaftskammer Oberelsass, Sainte Croix en Plaine; http://www.soel.de/beratung/ downloads/PPT $\% 20-\% 20$ Matthias $\% 20$ MEND $\% 20-\% 20$ Wirtschaftlichkeitsvergleich\%20-\%20D.pdf eingesehen am 21.10. 2014 (2013) 
W. Römmelt. Unruhe trotz Wachstum. Weinwirtschaft, 2/2015, 50-52 (2015)

Verband Deutscher Prädikatsweingüter. Zahlen, Daten, Fakten; http://www.vdp.de/de/der-vdp/zahlen -daten-fakten/ eingesehen am 12.10.2014 (2014)

B, Wechsler. Weinmarketing: Dienstleistungszentrum ländlicher Raum-Rheinland-Pfalz, Weinbaudomäne
Oppenheim. Intensivinterview am 11.11.2014 (2014)

H, Willer, M. Yussefi. The World of Organic Agriculture. Statistics and Emerging Trends; http://orgprints .org/5161/1/yussefi-2006overview.pdf, eingesehen am 14.10.2014 (2006) 